\title{
Closed Loop Navigation for Multiple Micro Robots
}

\author{
Grigoris Lionis, Dimos V. Dimarogonas and Kostas J. Kyriakopoulos
}

\begin{abstract}
Micro robotic agents are more difficult to control than 'macro' robots as they suffer from a number of inherit limitations.In this work we apply a previous result on decentralized navigation to the case of micro robots. The micro-robots we are interested in, move like unicycles with bounded turning radius. To control these micro-robots, we use a multi-level control strategy. In the upper level the agents are thought as omnidirectional. The middle layer maps these movements into the motion of a unicycle, and finally the lower level drive controls the micro-agent. We present simulations to demonstrate the validity of this approach.
\end{abstract}

\section{INTRODUCTION}

For the past few years, a number of researches have been studying aspects of micro robotics behavior, i.e. robotics on micro-scales. Robotics in these scales is often not a simple use of well studied principles and ideas from traditional "macro" robotics, but instead, new ideas, paradigms and tools have to be used, in order to cope with the behavior of robots in these scales.

In this work we will focus on the control of a micro robot on which motion constraints are imposed, due to the nature of the locomotion system. Specifically, we are trying to utilize the literature tools developed for groups of "macro robots" in the micro-world, by dividing the system into multiple levels, and by "isolating" the micro-effects from the higher levels, where the system can be thought of as a "macro" robotics system.

In general, micro locomotion systems are not as versatile as locomotion systems found on larger robots, but instead are constrained by a number of reasons including

- Power Constraints: Simultaneous rotational and linear movement could be prohibited by the power available

- Computational Constraints: Calculations needed for a micro-hexapod to rotate and translate simultaneous could exceed the on board resources

- Locomotion Structure: The locomotion system may not allow complex motion patterns

Our work is motivated by a class of cutting-edge multilegged micro-robots [4]. In these robots, the structure of the

This work is partially supported by the European Commission through contract FP6 IST 2002507006 ISWARM: Intelligent Small World Autonomous Robots for Micro-Manipulation, and by the Greek State Scholarship Foundation, IKY

G. Lionis is a PhD student with the department of Mechanical Engineering, National Technical Unversity of Athens, Zographou Greece glionemail.ntua.gr

D. Dimarogonas is a PhD student with the department of Mechanical Engineering, National Technical Unversity of Athens, Zographou Greece ddimar@mail.ntua.gr

K. Kyriakopoulos is with Faculty of Mechanical Engineering, National Technical Unversity of Athens, Zographou Greece kkyria@mail.ntua.gr locomotion systems does not allow the micro-robot to move arbitrarily, but instead it can move forwards and backwards but cannot turn arbitrarily, but only with a specified turning radius. This system moves roughly as a tricycle in which the steering wheel is locked in two positions, corresponding to the state in which the system moves forwards or backwards and to the state where the system rotates around a constant center of rotation located at a distance $\rho$ from the robot.

Control of multiagent robotic systems has flourished during the past few years. Control problems discussed by various researchers range from point to point collision free navigation of multiple robots, to flocking and swarming of mobile agents. In [6] the authors study the problem of centralized navigation of a multi-agent system consisting of omni-directional problems, while the same problem for unicycles is discussed in [7]. Flocking for mobile agents is also discussed by numerous researches including [10],[8],[3] . Most of this work on multirobot control is accomplished assuming robot kinematics that are either fully actuated, or correspond to a unicycle. The complex motion patterns associated with a micro robot, that in our case can be modeled as a unicycle that moves on a finite set of curvature trajectories, has not been examined in the literature.

On the other hand, the problem of bounded curvature vehicles has received attention, without any attempts to extend such work in the case of multi-agent control. In [1] the authors study the problem of stabilizing a kinematic unicycle on the plane, assuming that the robot moves with bounded curvature and that few sensory information are available to the robot. They propose a hybrid control technique that stabilizes the robot to a large class of trajectories. Their model assumes a robotic vehicle that can only move forwards, and therefore is not suitable to a robot that can move both forwards and backwards, since that would be a gross oversimplification of the actual robot capabilities. Moreover, their technique results in a relatively complex hybrid system, not easily implemented onto a computationally inefficient micro-robot.

In [9], the authors study the problem of calculating optimal paths for robots moving on bounded curvature trajectories, with the robot being able to move both forwards and backwards. The authors give a procedure that, for any given endpoints on a plane free of obstacles, will generate a small number of trajectories, in which the optimal trajectory belongs. Thus, by comparing these trajectories -either by brute force, or by applying some prefilters to reduce the costthe optimal one can be found. This result, solves completely the path planning problem, but is difficult to extend it in closed loop control and/or to multi agent systems. 
We could summarize the contribution of this paper, as a first attempt to utilize a controller developed for vehicles that move in the traditional "macro-robot" way, i.e. for vehicles exhibiting simple kinematics, to a class of micro robots exhibiting more complex kinematics. This approach has the advantage of being able to immediately use the vast literature on multi agent control.

\section{Problem Statement}

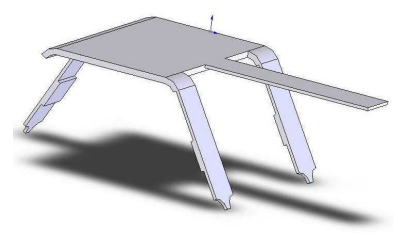

Fig. 1. ISWARM [4] microrobot

We have a group of $\mathscr{N}$ micro-robots moving on the plane. The state of each vehicle is given by

$$
q=\left[\begin{array}{l}
x \\
y \\
\theta
\end{array}\right]
$$

where $(x, y)$ are the Cartesian coordinates of the center of the robot w.r.t a global coordinate frame and $\theta$ is the orientation of the robot w.r.t. to the same coordinate frame. The kinematics of the vehicle are given by (2)

$$
\begin{gathered}
\dot{q}=V \cdot\left[\begin{array}{c}
\cos \theta \\
\sin \theta \\
\rho
\end{array}\right] \\
V \in\left[-V_{\text {max }},-V_{\text {mix }}\right] \cup 0 \cup\left[V_{\text {min }}, V_{\text {max }}\right], \rho \in\left\{-\rho_{0}, 0, \rho_{0}\right\}
\end{gathered}
$$

So the vehicle can either move forwards or backwards (w.r.t. its local position) or can rotate with a fixed turning radius (which is equal to $1 / \rho_{0}$ ). This system, corresponds to the kinematics of state of the art micro-robots (Fig. 1). Rotation of the robot is accomplished by rotating around the end point of one of the front legs, and therefore the motion envelope contains "self motions" with the curvature taking discrete values. Moreover, this system model, can be used to model tricycles, where the steering wheel is not free to rotate $360^{\circ}$. In this context, a number of automobile like vehicles, is described by equations of the form 2, when the turning wheel constraints are taken into acount.

The problem is to find a control law, that steers the microrobot from the initial positions $q_{i}^{\text {initial }}$ to the final positions $q_{i}^{\text {final }}$, while ensuring that their trajectories will be collision free, i.e.

$$
\left\|q_{i}-q_{j}\right\|_{p} \geq D
$$

where

$$
\|(x, y, \theta)\|_{p} \equiv|x|+|y|
$$

( $D$ is chosen large enough so that the actual micro-robots do not intersect i.e.

$$
D \geq 2 \cdot r+\delta
$$

where $r$ is the radius of the micro-robot).

\section{A. MicroRobot Communication-Sensing}

We will assume that each microrobot is equipped with a communication system capable of exchanging information within a communication radius $D_{c}>D$. We will not be concerned with bandwidth limitations in the communication system, partially because we are interested in exchanging a small amount of information within the micro-robotic network.

Finally, we assume that the microrobots are equipped with a sensing system, capable of measuring the state of all other micro-robots, within a sensing range $D_{s}$, with $D_{c}>D_{s}$.

The communication and sensing systems could, in principle, be interweaved, in the sense that sensing -of another vehicle in proximity- could be implemented by inter-agent communication.

\section{COMMUNICATION GRAPH}

We can define the communication graph $\mathscr{G}$ of the micro agent group, by defining its Adjacency matrix as

$$
M(i, j)=\left\{\begin{array}{l}
1,\left\|q_{i}-q_{j}\right\|_{p}<D_{c} \\
0,\left\|g_{i}-q_{j}\right\|_{p} \geq D_{C}
\end{array}\right.
$$

The communication graph of the micro-agent group is naturally decomposed on its connected components, i.e.

$$
\mathscr{G}=\mathscr{G}_{1} \cup \mathscr{G}_{2} \cup \ldots
$$

We state as an assumption necessary for the subsequent controller synthesis, that at every time instant, all agents belonging in the same connected component of the communication graph, share -through their communication systemthe same reference velocity, $\tilde{V}(i, t)$. The reference velocity is used as the rescaling value for the agent velocity. The micro robot cannot move with the velocity instructed by the holonomic controller, due to the structure of the actuators. The velocities of all the connected agents are rescalled with $\tilde{V}$ so that the final velocities value are within the actuators capabilities.

$$
\tilde{V}(i, t)=\tilde{V}(j, t) \forall t, \forall i, j s . t . \exists k: i, j \in \mathscr{G}_{k}
$$

At this point we are not explicitly defining the actual value of this virtual velocity, nor how it is evaluated at each time instant. We merely state the fact, that it is shared between all the agents on the same connected component.

\section{Multi-Level Control Scheme}

The proposed control scheme is depicted in Fig. 2. The decentralized navigation is accomplished using a "holonomic" controller. The agents are thought as being able to move omnidirectionally.

The velocity output of this controller is fed to the second level, which translates the velocity commands to linear and rotational velocities. We will explain in a subsequent paragraph the details of this transformation, and how it can be used in our case.

Finally, the non-holonomic velocity pair is fed to the micro-robotic drive, which regulates the switching frequency between the micro-robot vector fields, accomplishing thus the 
motion of the robot. The actual linear velocity of the robot is transmitted to all the robots in the neighborhood.

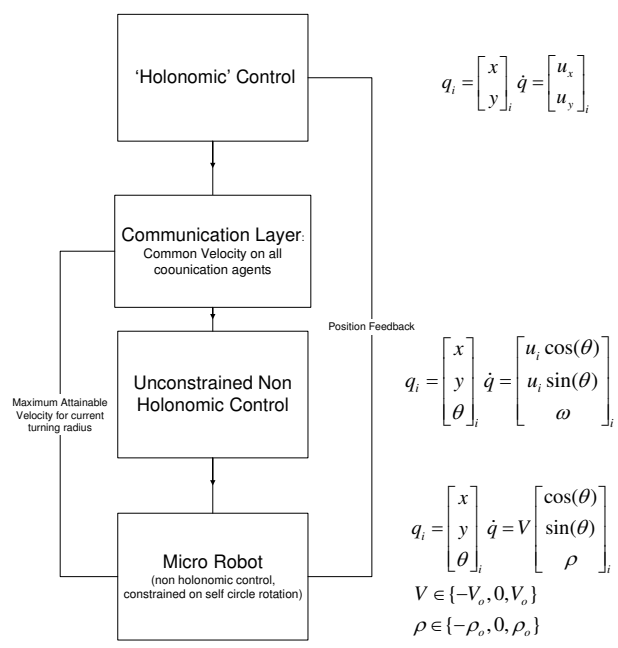

Fig. 2. Multi Level Control Scheme

\section{MultiAgent “Holonomic” Control}

We will use as the basic building block for our controller, the controller presented by Dimarogonas and Kyriakopoulos in [2]. In that work, the authors presented a decentralized controller, for solving the multi-agent movement problem for a group of agents, satisfying kinematics of the form

$$
\dot{q}=u
$$

The authors assume a sensing zone in which each agent can measure the exact position of all other agents.

The control law for each robot takes the form

$$
u_{i}=-K_{i} \frac{\partial \phi_{i}}{\partial q_{i}}
$$

where $\phi_{i}$ is specially constructed decentralized navigation function which encodes the information necessary for the robot's task, i.e. the position of the goal point, the positions of other robots within the sensing region etc. The navigation function is constructed as

$$
\phi_{i}=\frac{\gamma_{d i}+f_{i}}{\left.\left.\left(\left(\gamma_{d i}+f_{i}\right)^{k}+G_{i}\right)^{1 / k}\right)\right)}
$$

with $\gamma_{d i}=\left\|q-q_{i}^{\text {final }}\right\|$ the distance from the goal, $f_{i}$ a function ensuring that the robot will be able to move even after it has reached its target, $k$ a suitable large exponent, guaranteeing that the construction renders the closed loop system asymptotically stable, and $G_{i}$ the function that encodes the presence of other robots -obstacles- that have to be avoided.

Function $G_{i}$ is constructed by evaluating all the possible agent collision schemes in which agent $i$ is directly involved. These are decomposed into two agent collisions called "binary relations". For each binary relation -representing a collision between agent $i$ and another agent $j$ - an associated proximity function is calculated, having the form

$$
\beta_{i j}=\left\{\begin{array}{c}
\left\|q_{i}-q_{j}\right\|^{2}-\left(r_{i}+r_{j}\right)^{2},\left\|q_{i}-q_{j}\right\| \leq d_{C} \\
d_{C}^{2}-\left(r_{i}+r_{j}\right)^{2},\left\|q_{i}-q_{j}\right\|>d_{C}
\end{array}\right.
$$

In [2] the authors show, using previous results and tools from non-smooth analysis, that the complete construction is indeed a decentralized navigation function, i.e. that it converges asymptotically almost everywhere. ${ }^{1}$ Since the perception of another robot is via communication, it follows that the only constraint when choosing $d_{c}$ is

$$
d_{c} \leq D_{c}
$$

For a specific technical reason, $d_{c}$ has to be dynamic and pairwise (i.e. $d_{c}$ for different for each robot pair, and moreover it is not constant with time) and is therefore set to a value strictly less than $D_{c}$, i.e.

$$
d_{c}^{i j}<D_{c} .
$$

\section{HolONOMIC TO NON-HOLONOMIC}

In this section, we will show that it is possible for a nonholonomic system of the form

$$
\begin{gathered}
p=[q, \theta]=\left[\begin{array}{lll}
x_{1} & x_{2} & x_{3}
\end{array}\right] \\
\dot{q}=\left[\begin{array}{cc}
\cos \left(x_{3}\right) & 0 \\
\sin \left(x_{3}\right) & 0 \\
0 & 1
\end{array}\right]\left[\begin{array}{l}
u_{1} \\
u_{2}
\end{array}\right]
\end{gathered}
$$

to match the trajectories generated from a 'holonomic' system of the form

$$
\begin{gathered}
\tilde{q}=\left[\begin{array}{ll}
\tilde{x_{1}} & \tilde{x_{2}}
\end{array}\right] \\
\dot{\tilde{q}}=\tilde{u}
\end{gathered}
$$

provided that

$$
\|\dot{\tilde{q}}\| \neq 0
$$

By saying match the trajectories, we mean that given proper initial conditions for the holonomic and the non-holonomic system, we can compute an appropriate input for the holonomic system so that its trajectory matches the trajectory of the non-holonomic system. Formally, given that

$$
\begin{aligned}
q_{1}(0) & =\tilde{q}_{1}(0) \\
q_{2}(0) & =\tilde{q}_{2}(0) \\
p_{3}(0)=\theta(0) & =\arctan \left(\tilde{u}_{2}(0) / \tilde{u}_{1}(0)\right.
\end{aligned}
$$

then

$$
\forall \tilde{u} . s . t . \forall t \tilde{u}(t) \neq 0 \exists u(t) s . t . q(t)=\tilde{q}(t), \forall t .
$$

The subscript refer to components of the states. The sought control input is constructed using the transformation

$$
\begin{gathered}
u_{1}=\|\dot{\tilde{q}}\| \\
u_{2}=\tan ^{-1} \frac{\tilde{u}_{2} \dot{\tilde{q}}_{1}-\tilde{u}_{1} \dot{\tilde{q}}_{2}}{u_{1}}
\end{gathered}
$$

\footnotetext{
${ }^{1}$ Except a set of initial conditions with zero measure, that leads to saddle points
} 
It is straightforward to check that by using these control inputs the behavior of the holonomic and of the nonholonomic system are equivalent.

The control output of the multiagent controller described in the previous section, is smooth -hence differentiablealmost everywhere, and hence is trivial to render it in the form of eq. (6). Moreover, as the multiagent controller has navigation function properties, the output velocity of the multiagent controller can never be zero, except at the desired configurations.

A problem arises at points where

$$
\left\|q_{i}-q_{j}\right\|^{2}-\left(r_{i}+r_{j}\right)^{2}=d_{C}
$$

at the points where robot $i$ and robot $j$ begin to understand each other. In these points, the velocity of agents $i, j$ is not smooth -in fact the velocity might not even be continuous, and hence the conditions necessary to map the control of the holonomic system to the non-holonomic, do not hold. We circumvent this problem, using the fact that this discontinuity in the velocity is pointwise.

Specifically,assume that at time $t_{0}$ the distance between agents $i, j$ becomes $d_{C}$

$$
d_{i j}\left(t_{0}\right)=d_{C}
$$

and therefore at this time instant, the transformation breaks down.

We define as

$$
\begin{aligned}
& \theta_{t_{0}}^{-}=\lim _{t \rightarrow t_{0}^{-}} \tan ^{-1} \frac{\tilde{\dot{u}}_{2}(t)}{\tilde{\dot{u}}_{1}(t)} \\
& \theta_{t_{0}}^{+}=\lim _{t \rightarrow t_{0}^{+}} \tan ^{-1} \frac{\tilde{\dot{u}}_{2}(t)}{\tilde{\dot{u}}_{1}(t)}
\end{aligned}
$$

By assuming that the time set in which condition (9) is satisfied, is countable, (11) is well defined. By using this definition, we can augment the 'holonomic to non-holonomic' map, so that system (4) follows (8) when condition (9) is not satisfied, and when (9) is satisfied, system (4) undergoes an in-site rotation from $\theta_{t_{0}}^{-} \rightarrow \theta_{t_{0}}^{+}$.

With this way, our non-holonomic system can follow exactly the trajectory of the holonomic system, but in a different time frame, as the non-holonomic system has to undergo a countable number of rotations.

To ensure that the time instants on which (9) holds is countable we must discriminate between two cases.

The first case is when motion direction of the holonomic system after the switching point is not tangent to the switching surface. In this case, the requirement for countable switching points is trivially satisfied, as in this case the trajectory of the holonomic agent, after the switch will intersect the switching surface after a finite amount of time.

The second -and very rare- case is the case when the motion direction of the holonomic system after the switch is tangent to the switching surface. In this case we make the following adjustment. Agents $i$ and $j$ agree upon an increase on the value of their switching distance $d_{C}$, i.e.

$$
d_{C}^{i j} \leftarrow d_{C}^{i j}+\varepsilon
$$

This results that for a finite time the system will move without switches, and as a result the countability of the switches is satisfied. Moreover, the stability of the holonomic controller as described in [2] is not affected, as the exact value of the switching radius is irrelevant.

\section{Micro Robot Drive}

In a recent work [5], we have shown that the microrobot system we are examining can follow the trajectory of a unicycle, under ideal conditions of high bandwidth actuators. We base the construction of this open-loop driving on the idea of pulse width modulation.

\section{A. Discretization of the Control Inputs}

In the subsequent analysis, we will assume that the linear velocity of the robot belongs in $V \in\{V \max ,-V \max , 0\}$. By doing so we get results that are obviously compatible with system 2 , since we merely commit the control input to a discrete subset of original control input.

Moreover, in this case the control problem becomes a a purely discreet one. We have a number of vector fields on which the micro-robot can move on. In particular, in this case we can decompose the kinematics of the system into 4 vector fields, that cannot be simultaneously activated, and that characterize all the possible forward motion directions the robot, can achieve from a point in the state space. These vector fields, labeled $g_{1}, \ldots, g_{4}$ are the following

$$
\begin{gathered}
g_{1}=\left[\begin{array}{l}
0 \\
0 \\
0
\end{array}\right] \quad g_{2}=V \cdot\left[\begin{array}{c}
\cos \theta \\
\sin \theta \\
0
\end{array}\right] \\
g_{3}=V \cdot\left[\begin{array}{c}
\cos \theta \\
\sin \theta \\
\rho_{0}
\end{array}\right] \quad g_{4}=V \cdot\left[\begin{array}{c}
\cos \theta \\
\sin \theta \\
-\rho_{0}
\end{array}\right]
\end{gathered}
$$

Furthermore, the robot can move along v.f. $-g_{2},-g_{3},-g_{4}$, with the set of all these vector fields completely characterizing the system's available motion directions.

\section{B. Pule Width Modulation}

We propose to control this micro robot using pulse-width modulated control. It is intuitively obvious that if the robot swiftly alternates between two of these vector fields, its overall motion will lie somewhere between these two vector fields. So, by exploiting this fact, we can make the robot -by alternating continuously between 2 (or more) vector field, moves as a unicycle.

To make this a precise concept, we need a little notation. We define a switching function

$$
\hat{\sigma}(t, a, T)=\left\{\begin{array}{c}
0,0 \leq t<T \\
1, T \leq t<(a+1) T
\end{array}\right.
$$

and as

$$
\sigma(t, a, T)=\left\{\begin{array}{c}
\hat{\sigma}(t, a, T), 0 \leq t<(a+1) T \\
\hat{\sigma}(t-(a+1) T, a, T), t \geq(a+1) T
\end{array}\right.
$$

We define as $C_{g_{i}, g_{j}}^{a}\left(q_{0}\right)$ the solution of the differential equation

$$
\dot{q}=\sigma(t, a, T) g_{i}+(1-\sigma(t, a, T)) g_{j}, T \rightarrow 0
$$




\begin{tabular}{|c|c|}
\hline V.F. Comb. & Robots Behavior \\
\hline$g_{1}$ & Robot is Still \\
\hline$g_{2}$ & Robot Moves Straight \\
\hline$g_{3}$ & Robot Turns Right $r=1$ \\
\hline$g_{4}$ & Robot Turns Left $r=1$ \\
\hline$C_{g_{2}, g_{4}}^{a}$ & Robot Turns Forward Left $r=\frac{a}{a+1}$ \\
\hline$C_{-g_{2}, g_{4}}^{a}$ & Robot Turns Forward Left $r=-\frac{a}{a-1}$ \\
\hline
\end{tabular}

TABLE I

Summary of Vector Field ANd ASSOCIATEd Motion

The right hand of this differential equation, for any $T>$ 0 , is piecewise analytic, and therefore, for any $T>0$, the solution of the differential equation is well defined.

The motion of the micro-robot associated with v.f. $i, j$ with time constant $a$ is therefore $C_{g_{i}, g_{j}}^{a}$ Our control strategy lies in controlling the motion of the microrobot by constructing a suitable $C_{g_{i}, g_{j}}^{a}$, i.e. by choosing appropriately $i, j$ and $a$. Since we are interested in moving the micro-robot as a unicycle, we want to construct motion modes that move the system with arbitrary curvature.

We can prove the following : when we alternate between vector fields $g_{2}$ and $g_{3}$, on the limit of $T \rightarrow 0$, the robot will move along vector field

$$
g_{23 a}=V\left[\begin{array}{c}
\cos (\theta) \\
\sin (\theta) \\
\frac{a}{a+1}
\end{array}\right]
$$

, in the sense that the solution of 12 and the solution of 13 for the same initial condition $q_{0}$ will be the same $\forall t$

Formally, we can show that

$$
\begin{gathered}
C_{g_{2}, g_{4}}^{a} \sim V \cdot\left[\cos \theta \sin \theta-\frac{a}{a+1}\right]^{T} \\
C_{-g_{2}, g_{4}}^{a} \sim V^{*} \cdot\left[\cos \theta \sin \theta-\frac{a}{a-1}\right]^{T}
\end{gathered}
$$

with $\sim$ meaning that the produce the same trajectory, starting from the same initial conditions.

We can summarize some of the motion results when alternating between different v.f. ${ }^{2}$ in table I.

This driving scheme, enable the micro-robot, to follow an arbitrary path of a constraint free non-holonomic vehicle, in a different time scale, by choosing the vector field and the switching times.

\section{Velocity Considerations}

Care has to be taken as the velocity of the agents as given by the holonomic control scheme, cannot be trivially executed by the lower level controller.

Specifically, at a point of velocity non-differentiability, the linear velocity of the agent must become equal to zero. Moreover,when the micro-robot drive actuates the robots to a trajectory with smaller turning radius than its self turning radius, the velocity of the motion is necessarily decreased, as in this case the agent moves back and forward continuously.

\footnotetext{
${ }^{2}$ We do not present all the combinations, as all others are symmetrical with the ones on the table.
}

To cope with these problems we use the concept of the communication graph, as described in the previous sections. The rules with which the velocities of the agents are calculated are set as following

- When a robot has to rotate in place, the virtual velocity of all robots sharing the same component of the comm. graph is set to zero

- During motion, the velocities of the agents are set so that all are within reach of the micro-drive, and that the geometry of the multi-agent scheme is satisfied, in a different time scale

\section{Simulations}

To verify the validity of this approach, we tested this control framework in a simulated multi-agent scenario. The multiagent team consisted four microagents, designated it the pictures by their colors, arranged in an almost symmetrical configuration (Fig. 3) (the orientations of the micro-robots are not part of the desired final conditions)

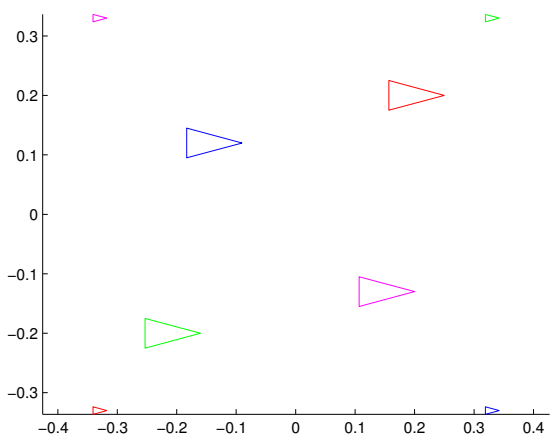

Fig. 3. Initial and final positions of the micro-robots

All the scales in the simulations are in decimeters. The velocity of the micro robot was set to $V=1 \mathrm{~mm} / \mathrm{sec}$ and $\omega=1 \mathrm{rad} / \mathrm{sec}$ to match expected values in real-world micro robots.

The result of the simulated run are depicted in Figures $(4,5,6,7)$. In Fig. 4, the robots begin moving towards their orientation. The first micro robots that come into contact are the blue and purple one. As a result all micro-robots stop moving -all robots are in communication range- and the blue,purple micro-robot begin to reorient themselves. In Phase II,III, Fig. 5 ,Fig.6 all robots are in contact and as a result all robots rotate to a new orientation. Finally, in Phase IV,Fig.7 the robots move towards their final destinations.

\section{CONCLUSIONS AND FUTURE WORKS}

In this paper the problem of concurrent movement of a group of micro-robots was examined. The micro robots are modeled as unicycles moving on constant curvature trajectories, and are assumed to have a communicationsensing system capable of local information sharing. The control method used was to map the velocity commands of the decentralized controller onto the motion directions of 


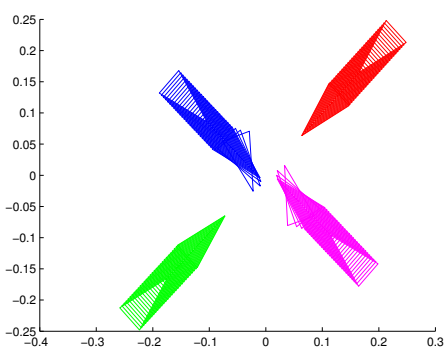

Fig. 4. Phase 1

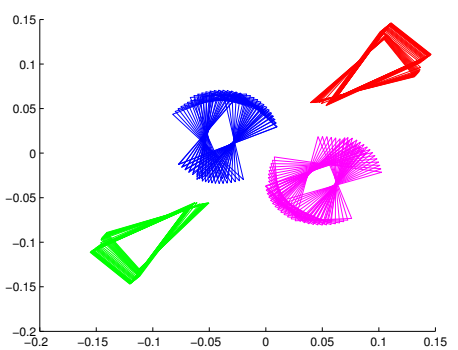

Fig. 5. Phase 2

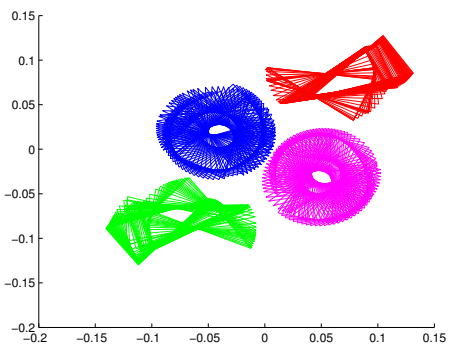

Fig. 6. Phase 3

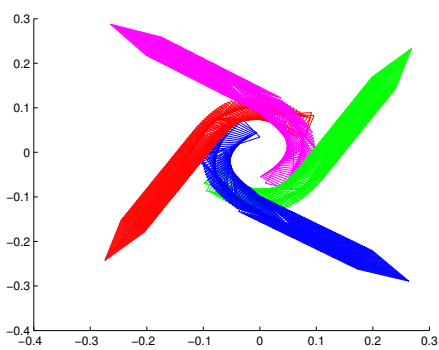

Fig. 7. Phase 4

the micro robots, in a way consistent with the kinematic capabilities of the robots. The high-level control of the micro-robots is the controller presented on [2], with suitable extensions for ensuring the feasibility of the whole scheme.

We presented simulated studies of this control technique to verify that the complete system behaves as stated, and to indicate that in practice, the necessary switching frequency is not infinite. The results demonstrate the validity of this approach, and points to an easy adaptation of various techniques for multi-agent control, to the case of micro-robots.

A point on this method that needs more attention is the micro-drive, and in particular the establishment of a closed loop control law that will map the non-holonomic motion commands to the micro-robot. This addition, will probably lead to a switching scheme that is provably correct even in the presence of noise, or in the presence of limited actuator bandwidth, but in this case the stability of the overall control law will have to be examined.

A second point worth of our attention is the smoothness of the control law. Had the control law of the holonomic system been smooth, then it would be much easier to map the holonomic motion of the micro-robot to a non-holonomic, eradicating the need for the $\varepsilon$ adjustment in the switching radius. A new decentralized navigation function structure, leading to smooth movement is necessary for this.

Finally, one could try to construct an optimal multirobot controller for robots moving with bounded curvature, based on the optimal control for a single robot. This controller would be probably discrete and complicated, as the single case is by itself complicated, but it would solve the problem much more 'economically' both mathematically -the path would be minimal- and physically -high frequency oscillations would be probably reduced-

\section{REFERENCES}

[1] A. Balluchi, A. Bicchi, and P. Souères. Path-following with a bounded-curvature vehicle: a hybrid control approach. International Journal of Control, 78(15):1228-1247, October 2005.

[2] D. V. Dimarogonas and K. J. Kyriakopoulos. Decentralized stabilization and collision avoidance of multiple air vehicles with limited sensing capabilities. 2005 American Control Conference, pages 46674772, 2005.

[3] Dimos V. Dimarogonas and Kostas J. Kyriakopoulos. Formation control and collision avoidance for multi-agent systems and a connection between formation infeasibility and flocking behavior. Proceedings 44th IEEE Conf. Decision and Control, 2005.

[4] Project ISWARM. http://microrobotics.ira.uka.de/.

[5] Grigoris Lionis and Kostas J. Kyriakopoulos. Pwm control for a microrobot moving on a discrete curvature trajectory set. Submitted to ICRA 2007., 2006.

[6] S. G. Loizou and K. J. Kyriakopoulos. Closed loop navigation for multiple holonomic vehicles. Proc. of IEEE/RSJ Int. Conf. on Intelligent Robots and Systems, pages 2861-2866, 2002.

[7] S.G. Loizou and K.J. Kyriakopoulos. Centralized feedback stabilization of multiple non-holonomic agents under input constraints. 5th IFAC Symposium on Intelligent Autonomous Vehicles, IAV 2004, 2004.

[8] Reza Olfati-Saber. Flocking for multi-agent dynamic systems: Algorithms and theory. IEEE Transactions on Automatic Control, 51(3), 2006.

[9] J. A. Reeds and L. A. Shepp. Optimal paths for a car that goes both forwards and backwards. Pacific Journal of Mathematics, 145(2):367393, 1990.

[10] Herbert G. Tanner, Ali Jadbabaie, and George J. Pappas. Stable flocking of mobile agents,. Proceedings 42th IEEE Conference on Decision and Control, pages 2016-2021, 2003. 